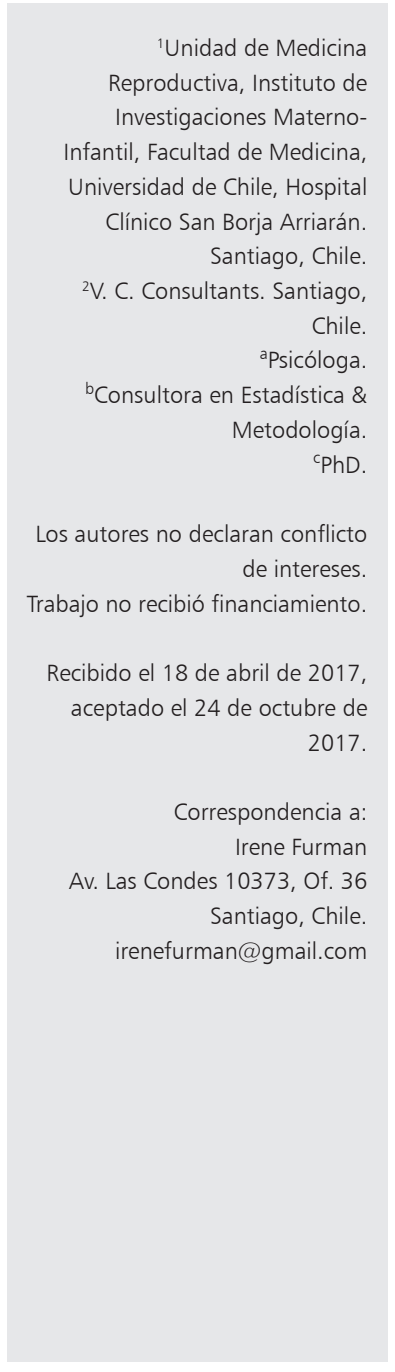

\section{Calidad de vida de parejas infértiles en el sector público de Chile}

\author{
IRENE FURMAN ${ }^{1, a, c}$, VENTURA CHARLIN ${ }^{2, b, c}$
}

\section{Quality of life of infertile couples in the public health sector in Chile}

Background: It is widely known that infertility and its treatment have a negative impact on patients' well-being, yet not much is known about patients' satisfaction with the quality of reproductive care. Purpose: To assess the quality of life (QoL) of patients receiving medical care under the Chilean public health system. Materials and Methods: The FertiQoL international questionnaire was applied to 260 patients (both sexes) referred from rural and urban areas to undergo in vitro fertilization at a major university public hospital. FertiQol evaluates QoL as a product of psychosocial well-being and treatment experience. Demographic and reproductive data were collected. Results: QoL due to treatment factors was significantly lower than psychosocial well-being. Couples from lower-density areas showed markedly worse medical satisfaction than patients treated in Chile's capital. No effects on QoL were observed as a function of age, education, or type of infertility. QoL was mostly affected by duration of infertility (inverse association) and geographical zone. Marital satisfaction was the component that most contributed to personal well-being. Conclusions: QoL decreased more due to extrinsic factors, particularly lack of access to reproductive care, poor organization of medical services, and deficient clinical interaction, than due to psychosocial distress. These findings call for a revision of the delivery of public fertility care to satisfy patients' needs and improve their QoL.

(Rev Med Chile 2017; 145: 1378-1386)

Key words: Chile; Hospitals, Public; Infertility; Quality of Life; Reproductive Medicine; Reproductive Health Services.

\section{$\mathrm{L}$} a infertilidad, definida como "la incapacidad de lograr un embarazo clínico después de 12 meses o más de relaciones sexuales no protegidas" 1 , afecta a $12-15 \%$ de la población chilena en edad reproductiva, estimándose que su incidencia aumente al postergar la maternidad persiguiendo mejores estándares personales y/o profesionales. Si bien la infertilidad no es incapacitante ni conlleva riesgo vital, genera daño psicológico sustancial al truncar un proyecto de vida medular a la pareja humana: tener un hijo ("la infertilidad no nos mata físicamente, pero nos mata el alma"). Se producen desajustes personales (ansiedad, culpas, baja autoestima/distorsión de la identidad de género secundarias a exigencias socioculturales), relacionales (conflictos de pareja, disfunciones sexuales) y sociales (estigmatización, desadaptación, aislación $)^{2-5}$. A lo anterior, se suma el desgaste derivado de los tratamientos, que son: invasivos de la intimidad y disruptivos del funcionamiento cotidiano, de resultados inciertos requiriendo iteración (ciclos esperanza/fracaso), y progresivos hacia mayor complejidad médica. Como resultante, las parejas exhiben una disminución del bienestar personal global y de la calidad de vida $(\mathrm{CV})^{6-9}$. La Organización Mundial de la Salud clasifica la infertilidad en el quinto lugar de la lista de moderate to severe disabilities in the global population under the age of $60^{10}$.

Investigaciones recientes señalan que varia- 
bles del sistema sanitario (e.g. acceso a atención, continuidad de las prestaciones, interacción con el equipo médico) afectan la CV de los usuarios y alteran la permanencia en los tratamientos ${ }^{11}$. Según un meta-análisis europeo ${ }^{12}$, 19\% de los casos de infertilidad abandonan los tratamientos por insatisfacción con aspectos organizacionales o relacionales, reduciendo sus posibilidades de embarazo.

Chile es uno de los pocos países del mundo que ha desarrollado guías nacionales para el tratamiento de la infertilidad definiendo protocolos por nivel de atención en la red asistencial (niveles primario, secundario, y terciario), otorgando evaluación y terapias de baja y alta complejidad ${ }^{13}$. El Instituto de Investigaciones Materno Infantil (IDIMI), Universidad de Chile/Hospital Clínico San Borja Arriarán, es desde 1992 el centro de referencia nacional para fertilización in vitro a parejas derivadas desde hospitales regionales y metropolitanos, el cual incluye atención psicológica especializada. Si bien a nivel nacional se ha multiplicado la cobertura clínica, no se ha investigado el nivel de bienestar de los/las pacientes, ni su satisfacción con los servicios estipulados. Resulta fundamental evaluar CV en los programas públicos para propiciar el enfoque humanitario centrado en los derechos de los/las pacientes ${ }^{14-16}$, junto a maximizar la adherencia a las indicaciones médicas y optimizar la eficiencia de los recursos. $\mathrm{Al}$ registrar la perspectiva de las parejas y su experiencia con los tratamientos, se logra identificar sus expectativas frente a los programas de salud y el grado de satisfacción con las prestaciones otorgadas, y finalmente evaluar la calidad de la gestión sanitaria. El objetivo del presente estudio fue evaluar la CV en los tratamientos de infertilidad de baja complejidad y su relación con variables demográficas y reproductivas en el sector público chileno.

\section{Material y Método}

\section{Participantes}

Doscientos sesenta pacientes (163 mujeres, 97 hombres), derivados desde centros regionales y de la Región Metropolitana (RM) al IDIMI. Completaron: (i) hoja de antecedentes (sexo, edad, educación, región de procedencia, tipo, duración, y causa de infertilidad) y (ii) cuestionario Fertility
Quality of Life Questionnaire (FertiQoL), versión en castellano?.

\section{Diseño}

Estudio transversal descriptivo cuantitativo, aprobado por el Comité de Ética del Servicio de Salud Metropolitano Central. Los datos se recolectaron en IDIMI entre enero-septiembre de 2016. El cuestionario fue administrado previo a la fertilización in vitro, por lo tanto recoge percepción retrospectiva de tratamientos de baja complejidad en los centros médicos de origen.

\section{Instrumento}

FertiQoL es un instrumento psicométrico desarrollado conjuntamente por las Sociedades Europea y Norteamericana de Medicina Reproductiva para medir CV específicamente asociada a la infertilidad y está validado internacionalmente ${ }^{9}$. Examina $\mathrm{CV}$ en dos ámbitos: nivel de bienestar personal y grado de satisfacción con aspectos médicos.

\section{Estructura del FertiQoL}

Consta de 2 escalas principales: a) Escala FertiQoL-Core que mide nivel de bienestar personal en 4 dimensiones o sub-escalas: Emocional, Mente-Cuerpo, Relacional, y Social (total 24 ítems), y b) Escala FertiQoL-Tratamiento compuesta por 2 sub-escalas: Tolerancia (grado de tolerancia a las molestias físicas de los tratamientos, 4 ítems) y Satisfacción (traducción libre del original Environment para facilitar comprensión del texto), que evalúa el grado de satisfacción con los servicios médicos y con la relación médico-paciente, 6 ítems). Se asigna una puntuación numérica entre 0 y 100 , en que mayor puntaje corresponde a mejor CV. Se evaluó la confiabilidad del FertiQoL para la muestra con el coeficiente alfa de Cronbach, obteniendo una buena consistencia interna en ambas escalas principales: 0,87 (FertiQoL-Core) y 0,79 (FertiQoL-Tratamiento).

\section{Análisis estadísticos}

Los resultados se reportaron como media y desviación estándar (DE) para las variables cuantitativas y porcentajes para las variables categóricas. Se analizaron las variables demográficas y reproductivas usando estadísticos descriptivos, utilizando la prueba de $\chi^{2}$ de asociación e independencia para comparar medias y proporciones. 
En el análisis de puntajes del FertiQol se utilizó la prueba t de Student para comparar dos muestras independientes y análisis de varianza (ANOVA) para más de 2 grupos. Las variables independientes fueron: sexo, edad, educación, procedencia, además de tipo, duración, y causa de la infertilidad. En las comparaciones múltiples, se corrigieron los valores de p utilizando el método de Tukey-Kramer o método HSD (Honestly Significant Difference) que emplea el estadístico de rango estudentizado para realizar todas las comparaciones por pares entre los grupos. Se consideraron estadísticamente significativos los valores de $\mathrm{p}$ inferiores a 0,05 . Todos los análisis estadísticos se realizaron en SAS 9.4@ para Windows.

\section{Resultados}

\section{Descripción de la muestra}

La Tabla 1 presenta los datos demográficos y reproductivos por región y totales de 260 pacientes (63\% mujeres, $37 \%$ hombres). La edad fluctuó entre 24 y 53 años con una media de 35,5; los pacientes de regiones eran mayores que los de RM (36,4 vs 34,8$)$. Un $58 \%$ de la muestra provenía de RM. El nivel educacional fue relativamente alto, con educación media completa en $85 \%$ de los participantes. La duración de la infertilidad fue más extensa en regiones que en $\mathrm{RM}(8,8$ vs 7,5 años). En regiones la causa de infertilidad más prevalente fue la femenina $(70 \%)$, mientras que

Tabla 1. Características demográficas y reproductivas por Procedencia y Total

\begin{tabular}{|c|c|c|c|}
\hline & $\begin{array}{c}\text { Santiago } \\
\text { n (\%) }\end{array}$ & $\begin{array}{c}\text { Regiones } \\
\text { n (\%) }\end{array}$ & $\begin{array}{c}\text { Total } \\
\text { n }(\%)\end{array}$ \\
\hline Participantes & 151 & 109 & $260(100)$ \\
\hline $\begin{array}{l}\text { Sexo } \\
\text { Mujeres } \\
\text { Hombres }\end{array}$ & $\begin{array}{l}84(55,6)^{* *} \\
67(44,4)^{* *}\end{array}$ & $\begin{array}{l}79(72,5)^{* *} \\
30(27,5)^{* *}\end{array}$ & $\begin{array}{r}163(62,7) \\
97(37,3)\end{array}$ \\
\hline $\begin{array}{l}\text { Edad } \\
\text { Promedio (DE) } \\
<35 \text { años } \\
35+\text { años }\end{array}$ & $\begin{array}{l}34,8(5,1)^{*} \\
67(44,4)^{*} \\
84(55,6)^{*}\end{array}$ & $\begin{array}{l}36,4(5,0)^{*} \\
38(34,9)^{*} \\
71(65,1)^{*}\end{array}$ & $\begin{array}{r}35,5(5,2) \\
105(40,4) \\
155(59,6)\end{array}$ \\
\hline $\begin{array}{l}\text { Procedencia } \\
\text { RM } \\
\text { Regiones }\end{array}$ & $\begin{array}{l}\text { NA } \\
\text { NA }\end{array}$ & $\begin{array}{l}\text { NA } \\
\text { NA }\end{array}$ & $\begin{array}{l}151(58,1) \\
109(41,9)\end{array}$ \\
\hline $\begin{array}{l}\text { Nivel educacional } \\
\text { Básico } \\
\text { Medio } \\
\text { Técnico-Universitario }\end{array}$ & $\begin{array}{l}18(11,9) \\
79(52,3) \\
54(35,8)\end{array}$ & $\begin{array}{l}18(16,5) \\
53(48,6) \\
38(34,9)\end{array}$ & $\begin{array}{r}36(13,8) \\
132(50,8) \\
92(35,4)\end{array}$ \\
\hline $\begin{array}{l}\text { Tipo de infertilidad } \\
\text { Primaria } \\
\text { Secundaria }\end{array}$ & $\begin{array}{r}103(68,2) \\
48(31,8)\end{array}$ & $\begin{array}{l}68(63,0) \\
40(37,0)\end{array}$ & $\begin{array}{r}171(66,0) \\
88(34,0)\end{array}$ \\
\hline $\begin{array}{l}\text { Duración de infertilidad } \\
\text { Promedio (DE) } \\
<6 \text { años } \\
6 \text { a } 9 \text { años } \\
10+\text { años }\end{array}$ & $\begin{array}{l}7,5(3,8)^{*} \\
59(39,1)^{*} \\
50(33,1) \\
42(27,8)^{*}\end{array}$ & $\begin{array}{l}8,8(4,6)^{*} \\
31(28,7)^{*} \\
35(32,4) \\
42(38,9)^{*}\end{array}$ & $\begin{array}{r}8,1(4,2) \\
90(34,8) \\
85(32,8) \\
84(32,4)\end{array}$ \\
\hline $\begin{array}{l}\text { Causa de infertilidad } \\
\text { Factor masculino } \\
\text { Factor femenino } \\
\text { Factor mixto } \\
\text { Factor desconocido }\end{array}$ & $\begin{array}{l}29(19,2) \\
60(39,8)^{* * *} \\
52(34,4)^{* * *} \\
10(6,6)\end{array}$ & $\begin{aligned} 19 & (17,6) \\
76 & (70,4)^{* * *} \\
7 & (6,5)^{* * *} \\
6 & (5,5)\end{aligned}$ & $\begin{array}{r}48(18,5) \\
136(52,5) \\
59(22,8) \\
16(6,2)\end{array}$ \\
\hline
\end{tabular}

${ }^{*} p<0,05 ;{ }^{* *} p<0,01 ; * * * p<0,001$. 
en RM se distribuyeron similarmente las causas femenina y mixta ( $40 \%$ y $34 \%$ ).

\section{Puntajes globales en cuestionario FertiQoL}

La Figura 1 resume los puntajes de CV en las distintas escalas y sub-escalas del FertiQol. Se observa una discrepancia notoria entre las dos escalas principales, con un promedio en FertiQoL-Tratamiento visiblemente inferior al promedio FertiQoL-Core (60,05 vs 71,43). Destaca una diferencia sustancial entre las dos sub-escalas que conforman Tratamiento, siendo la media en Satisfacción 23 puntos inferior a la media de Tolerancia $(50,67$ vs 73,69$)$. En contraste, las medias en las sub-escalas de Core fueron similares entre sí, excepto la sub-escala Relacional que tuvo significativamente mayor puntuación (78,63 Relacional, 69,29 Emocional, 69,82 Mente-Cuerpo, y 67,99 Social).

\section{Análisis específicos en FertiQol-Core}

La Tabla 2 muestra que hubo diferencias significativas en puntajes promedio en función de las variables Procedencia, Duración de la infertilidad, y Causa de la infertilidad: pacientes de regiones reportaron puntajes más altos que los de RM $(74,87$ vs 68,95$)$, pacientes con infertilidad entre 6-9 años obtuvieron un puntaje más bajo $(67,71)$ que los de otros rangos de tiempo, y pacientes con causa de infertilidad mixta alcanzaron peor puntaje $(66,82)$ que los pacientes con otras etiologías. Al analizar separadamente los puntajes de las sub-escalas Emocional, Mente-Cuerpo, Relacional y Social en función de las variables independientes (análisis no exhibidos, ver medias en Figura 1), se observó que el puntaje Relacional $(78,63)$ se mantuvo sistemáticamente más alto que los otros. La Tabla 3 muestra, a modo de ejemplo, el análisis de las sub-escalas comparadas por Procedencia; se advierte que en regiones las medias fueron consistentemente más altas que en RM, con excepción de las medias de Relacional que no difirieron; paralelamente se observa que en ambas procedencias la media Relacional fue superior a las otras tres.

\section{Análisis específicos en FertiQol-Satisfacción}

Dado que no se encontraron diferencias entre los puntajes de la escala Tolerancia (análisis no exhibido), se detallan los análisis de la escala Satisfacción. La Tabla 4 muestra diferencias significativas de puntajes en función de: Sexo (media masculina 55,99 vs media femenina 47,56), Duración de infertilidad (medias 56,62, 50,35 y 45,10 en rangos crecientes de duración), y Procedencia (pacientes de regiones obtuvieron un puntaje promedio 17 puntos más bajo que pacientes de RM, 40,95 vs $57,84)$. Es evidente entonces, que la procedencia geográfica fue la variable que generó la diferencia de mayor magnitud en FertiQol-Satisfacción. Se analizaron separadamente los ítems que conforman esta sub-escala. Los puntajes totales fueron, de menor a mayor: Disponibilidad de los Servicios (media 38,33; puntaje más bajo de todo el cues-

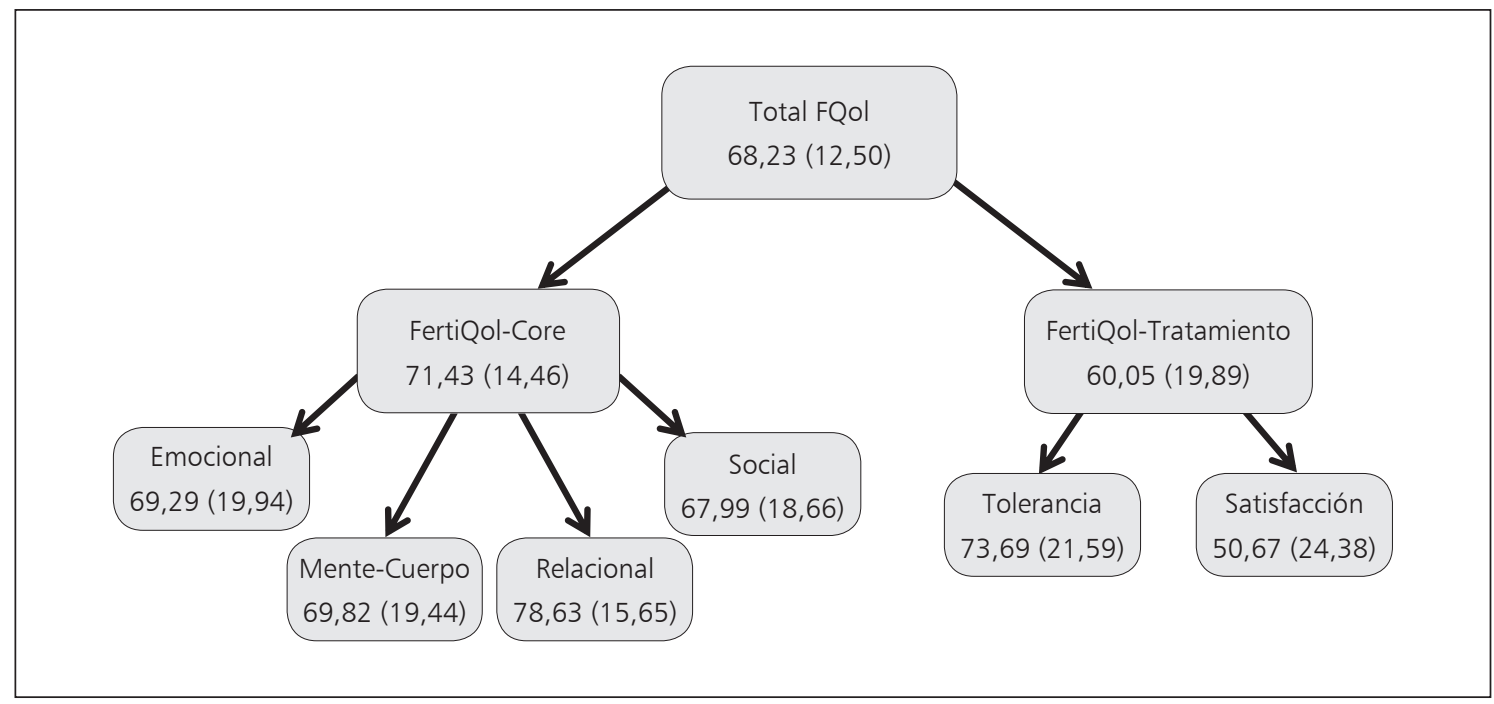

Figura 1. Esquema descriptivo de los puntajes en el cuestionario FertiQoL: medias (desviaciones estándar). 
Tabla 2. Puntajes Escala FertiQoL-Core por características demográficas y reproductivas

\begin{tabular}{|c|c|c|c|}
\hline & Media (DE) & $t(g l)$ & $\mathbf{p}$ \\
\hline FertiQoL-Core Total & $71,43(14,46)$ & & \\
\hline \multicolumn{4}{|l|}{ Sexo } \\
\hline Mujeres & $70,34(14,44)$ & $-1,58(258)$ & 0,1144 \\
\hline Hombres & $73,27(14,39)$ & & \\
\hline \multicolumn{4}{|l|}{ Edad } \\
\hline$<35$ años & $71,16(14,20)$ & $-0,25(258)$ & 0,8001 \\
\hline $35+$ años & $71,62(14,68)$ & & \\
\hline \multicolumn{4}{|l|}{ Procedencia } \\
\hline RM & $68,95(15,25)$ & $-3,42(258)$ & 0,0007 \\
\hline Regiones & $74,87(12,57)$ & & \\
\hline Nivel educacional & Media (DE) & $\mathbf{F}(\mathbf{g l})$ & $\mathbf{p}$ \\
\hline Básico & $68,57(13,88)$ & $1,68(2,257)$ & 0,1891 \\
\hline Medio & $70,84(15,34)$ & & \\
\hline Técnico-Universitario & $73,40(13,23)$ & & \\
\hline Tipo de infertilidad & Media (DE) & $t(g l)$ & $\mathbf{p}$ \\
\hline Primaria & $70,19(14,88)$ & $-1,81(257)$ & 0,0719 \\
\hline Secundaria & $73,60(13,29)$ & & \\
\hline Duración de infertilidad & Media (DE) & $\mathbf{F}(\mathbf{g l})$ & $\mathbf{p}$ \\
\hline$<6$ años & $73,69(15,30)$ & $4,34(2,256)$ & 0,0141 \\
\hline 6 a 9 años & $67,71(13,84)^{*}$ & & \\
\hline $10+$ años & $72,73(13,65)$ & & \\
\hline Causa de infertilidad & Media (DE) & $\mathbf{F}(\mathbf{g l})$ & $\mathbf{p}$ \\
\hline Factor masculino & $74,32(12,76)$ & $2,92(3,255)$ & 0,0346 \\
\hline Factor femenino & $72,24(15,10)$ & & \\
\hline Factor mixto & $66,82(13,83)^{*}$ & & \\
\hline Factor desconocido & $72,26(13,67)$ & & \\
\hline
\end{tabular}

Tabla 3. Puntajes Escala FertiQoL-Core y Sub-escalas comparadas por Procedencia

\begin{tabular}{|lcccc|}
\hline & RM media (DE) & Regiones media (DE) & t (gl) & p \\
\hline Sub-escala Emocional & $65,76(21,12)$ & $74,18(17,10)$ & $-3,43(255)$ & 0,0005 \\
\hline Sub-escala Mente-cuerpo & $67,22(20,67)$ & $73,43(17,04)$ & $-2,65(255)$ & 0,0087 \\
\hline Sub-escala Relacional & $77,17(15,50)$ & $80,65(15,71)$ & $-1,78(258)$ & 0,0769 \\
\hline Sub-escala Social & $65,73(19,26)$ & $71,12(17,40)$ & $-2,32(258)$ & 0,0213 \\
\hline Total escala Core & $68,96(15,25)$ & $74,87(12,57)$ & $-3,42(255)$ & 0,0007 \\
\hline
\end{tabular}

tionario), Empatía por parte del personal médico $(51,40)$, Comunicación por parte del personal médico $(58,17)$ y Calidad de los Procedimientos $(58,61)$. La Figura 2 muestra los puntajes separados por Procedencia. Se destaca que el orden de los puntajes fue uniforme en ambas procedencias. Las diferencias intra-ítem fueron todas significativas, siendo Disponibilidad de los servicios la más marcada (24,55 puntos) y Empatía la menos pronunciada (10,10 puntos). 
Tabla 4. Puntajes sub-escala Satisfacción por características demográficas y reproductivas

\begin{tabular}{|c|c|c|c|}
\hline & Media (DE) & $t(g l)$ & p \\
\hline Sub-escala Satisfacción (total) & $50,67(24,38)$ & & \\
\hline \multicolumn{4}{|l|}{ Sexo } \\
\hline Mujeres & $47,56(23,34)$ & \multirow{2}{*}{$-2,71(255)$} & \multirow[b]{2}{*}{0,0072} \\
\hline Hombres & $55,99(25,31)$ & & \\
\hline \multicolumn{4}{|l|}{ Edad } \\
\hline$<35$ años & $54,16(25,38)$ & \multirow{2}{*}{$-1,87(255)$} & \multirow[b]{2}{*}{0,0630} \\
\hline $35+$ años & $48,38(23,51)$ & & \\
\hline \multicolumn{4}{|l|}{ Procedencia } \\
\hline $\mathrm{RM}$ & $57,84(21,60)$ & \multirow[b]{2}{*}{$-5,83(255)$} & \multirow[b]{2}{*}{$<0,0001$} \\
\hline Regiones & $40,95(24,67)$ & & \\
\hline Nivel educacional & Media (DE) & $\mathbf{F}(\mathbf{g l})$ & $\mathbf{p}$ \\
\hline Básico & $45,14(25,99)$ & \multirow{3}{*}{$1,05(2,254)$} & \multirow{3}{*}{0,3526} \\
\hline Medio & $51,43(24,63)$ & & \\
\hline Técnico-Universitario & $51,71(23,35)$ & & \\
\hline Tipo de infertilidad & Media (DE) & $t(g l)$ & $\mathbf{p}$ \\
\hline Primaria & $49,77(24,27)$ & \multirow{2}{*}{$-0,77(254)$} & \multirow{2}{*}{0,4419} \\
\hline Secundaria & $52,25(24,73)$ & & \\
\hline Duración de la infertilidad & Media (DE) & $\mathbf{F}(\mathbf{g l})$ & $\mathbf{p}$ \\
\hline$<6$ años & $56,62(23,35)$ & \multirow{3}{*}{$4,93(2,253)$} & \multirow{3}{*}{0,0079} \\
\hline 6 a 9 años & $50,35(21,68)$ & & \\
\hline $10+$ años & $45,10(26,81)^{*}$ & & \\
\hline Causa de la infertilidad & Media (DE) & $\mathbf{F}(\mathbf{g l})$ & $\mathbf{p}$ \\
\hline Factor masculino & $51,54(23,81)$ & \multirow{4}{*}{$1,99(3,252)$} & \multirow{4}{*}{0,1154} \\
\hline Factor femenino & $47,70(25,10)$ & & \\
\hline Factor mixto & $55,14(23,08)$ & & \\
\hline Factor desconocido & $58,96(21,16)$ & & \\
\hline
\end{tabular}

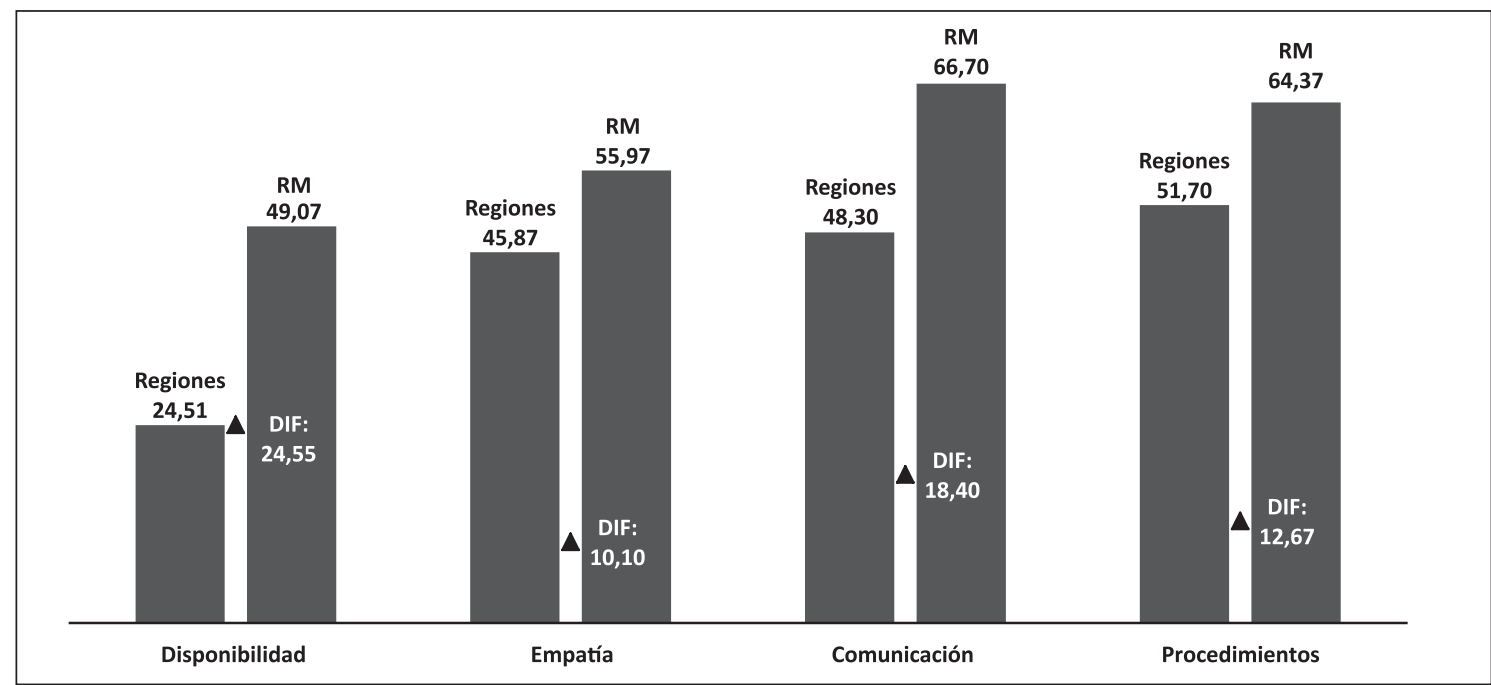

Figura 2. Medias y diferencias de puntajes en ítems de Sub-Escala Satisfacción por Procedencia*. Disponibilidad de Servicios incluye ítems 2 y 7 y Comunicación incluye ítems 9 y 10. 


\section{Discusión}

Este estudio investigó la $\mathrm{CV}$ de pacientes infértiles usuarios de atención en salud reproductiva conforme al protocolo establecido en la red asistencial del Ministerio de Salud ${ }^{13}$. Se evaluó la variación de $\mathrm{CV}$ en función de factores moderadores previo a iniciar tratamiento in vitro, considerando: 1) el impacto psicosocial generado por la infertilidad, y 2) la satisfacción de los pacientes con los servicios recibidos. Como instrumento psicométrico se utilizó FertiQoL, actual gold standard internacional ${ }^{9}$.

\section{Impacto psicosocial de la infertilidad}

La evaluación en este aspecto fue similar a la de poblaciones europeas ${ }^{9,17-22}$, aunque la variabilidad de muestreo no permite comparaciones exactas. La percepción de las parejas sobre el impacto de la infertilidad en su vida personal se mantuvo independientemente del sexo, edad, nivel educacional, y tipo de infertilidad. Pacientes de regiones expresaron mayor bienestar personal que los de la Región Metropolitana. Por otra parte, la CV fue menor durante el rango intermedio de infertilidad (6-9 años), sugiriendo que la reacción emocional a esta enfermedad es dinámica en el tiempo, con períodos de vulnerabilidad personal variable, aunque tiende hacia mayor equilibrio emocional y adaptación psicosocial ${ }^{4,22}$, en concordancia con las observaciones clínicas ("uno va madurando este proceso", "una se hace más fuerte", "uno aprende a enfrentar la presión social”). Asimismo, es notable el hallazgo que los/las pacientes atribuyeron sistemáticamente su mayor bienestar a la relación de pareja, sustentando la evidencia de distintos países ${ }^{19,20,23,24}$, sugiriendo que la satisfacción marital sería una tendencia transcultural. Efectivamente en la práctica clínica se advierte la resiliencia de la pareja infértil y su capacidad de sobreponerse a las dificultades afectivas y relacionales en la búsqueda conjunta del hijo ("el sufrimiento nos ha unido aún más", "queremos un hijo en común, no con otra persona fértil"). Por último, influyó en el bienestar personal la causa de la infertilidad, siendo la etiología mixta (ambas personas presentan disfunciones reproductivas) la que provocó mayor detrimento. Estos hallazgos tienen aplicabilidad clínica directa, pues identifican a los pacientes de mayor fragilidad emocional que se beneficiarían de atención psicológica focalizada e integrada al tratamiento reproductivo.

\section{Satisfacción con los servicios de medicina reproductiva}

La CV asociada a los aspectos médicos fue notoriamente inferior a la CV personal. Esta baja no se atribuyó a las molestias físicas de los procedimientos, sino a una insatisfacción sustancial con la calidad de los servicios reproductivos, específicamente con i) la pobre disponibilidad de los servicios; ii) la deficiente empatía y habilidad comunicacional por parte del personal médico. $\mathrm{La}$ insatisfacción de las parejas se mantuvo independientemente de la edad, nivel educacional, tipo de infertilidad, y causa de infertilidad. Las pacientes mujeres mostraron mayor insatisfacción, concordando con el grueso de las publicaciones, en que las pacientes reportan una experiencia más negativa de la infertilidad que sus contrapartes masculinos ${ }^{25-28}$. Sin duda, influye el que sean las mujeres quienes experimentan en su cuerpo la casi totalidad de los procedimientos médicos (independientemente de la etiología reproductiva), sufriendo mayor desgaste físico y psicológico, además de experimentar la llegada de la menstruación como señal ineludible del fracaso reproductivo. También repercutió en la satisfacción de los usuarios la duración de la infertilidad, en sentido lineal inverso (la evaluación empeoró a medida que se alargó la infertilidad). Sin embargo, la mayor discrepancia en CV se debió a la procedencia geográfica:

\section{Satisfacción de los usuarios en función de la procedencia}

Las parejas de regiones expresaron ostensiblemente mayor insatisfacción con los servicios reproductivos que aquellas de la RM. Comparaciones en profundidad identificaron que la mayor disconformidad fue con la pobre disponibilidad de atención especializada, evaluada 24,55 puntos más baja que en RM (Figura 2), seguida de la disconformidad con la deficiente empatía y comunicación por parte del personal médico, y luego con la calidad de los procedimientos. Es interesante notar que, si bien la insatisfacción fue mayor en regiones, el patrón de descontento se mantuvo uniforme para ambas procedencias (Figura 2), sugiriendo que la atención en infertilidad adolecería de las mismas falencias a nivel nacional. 
En conclusión, este estudio evaluó distintos componentes de CV utilizando un mismo instrumento psicométrico. Hubo evidencia inequívoca de que la mayor baja en CV proviene de factores extrínsecos (médicos y organizacionales) más que de desajustes psicosociales. Este hallazgo fue inesperado ya que la impresión generalizada entre clínicos e investigadores ${ }^{29-31}$ es que la principal dificultad de los pacientes infértiles radica en factores intrapsíquicos (e.g., niveles de ansiedad, rasgos de carácter, dificultades de pareja). Estos resultados validan propuestas recientes que plantean que la $\mathrm{CV}$ en infertilidad es un constructo multideterminado que no debiera ignorar el rol de factores externos y ajenos al dominio de los pacientes ${ }^{11,12,32}$. La calidad de la gestión sanitaria y la atención brindada, por ejemplo, incidirían en el estado emocional de los/las pacientes, con un detrimento del bienestar global. Se advirtió paralelamente que la disconformidad de las parejas fue aún más pronunciada en regiones. Estos resultados son inéditos y señalan que las necesidades que más apremian a las parejas en las etapas de diagnóstico y tratamiento de baja complejidad son tener un acceso más oportuno a medicina reproductiva especializada en infertilidad y una interacción clínica de mejor calidad. Esta información es útil para optimizar la eficiencia de la atención en salud reproductiva, pues sitúa las áreas específicas que requieren mejoramiento. Como sugerencias directas, se plantea perfeccionar los mecanismos de derivación regional a centros especializados, y capacitar a los profesionales de salud en habilidades comunicacionales y de empatía con la vivencia del paciente ("debieran hacerles ver a los médicos que uno no es una estadística, sino una persona que está sufriendo", "debieran enseñarle a los doctores a que no nos enfermen más que la enfermedad"). Tales medidas, aparte de incrementar la CV de los/las pacientes, deberían redundar en mayor adherencia terapéutica y en mejores resultados clínicos, optimizando así los recursos.

Agradecimientos: Las autoras agradecen especialmente al Dr. Pablo Céspedes P. (Director Programa de Reproducción Asistida, Instituto de Investigaciones Materno Infantil, Universidad de Chile) por su constante interés y dedicación a promover la calidad de vida de las parejas infértiles, y a la Dra. Paulina Troncoso E. (Facultad de Medicina, Universidad de Chile, Encargada Pro- grama Nacional Salud de la Mujer, Ministerio de Salud) por su generosa contribución a la versión final del artículo.

\section{Referencias}

1. Zegers-Hochschild F, Adamson GD, de Mouzon J, et al. International Committee for Monitoring Assisted Reproductive Technology; World Health Organization (ICMART) and the World Health Organization (WHO) revised glossary of ART terminology, 2009. Hum Reprod 2009; 24 (11): 2683-7.

2. Mahlstedt $P$. The psychological component of infertility. Fertil Steril 1985; 45 (3): 335-46.

3. Furman I, Devoto L, Ramos A, Urmeneta L, Vantman D, Fuentes A. Estudio comparativo de los efectos psicológicos de la infertilidad en hombres y mujeres chilenos. Rev Chil Obstet Ginecol 1997; 62 (1): 9-14.

4. Covington S, Burns LH. Infertility counseling: a comprehensive handbook for clinicians. $2^{\text {nd }}$ edition. New York: Cambridge University Press 2006.

5. Furman I. Aspectos Psicosociales de la infertilidad. En Pérez Sánchez, A, Editor, Ginecología, Santiago, Chile: Editorial Mediterráneo Ltda. 2014, p. 743-53.

6. Domar A, Zuttermeister P, Friedman R. The psychological impact of infertility: a comparison with patients with other medical conditions. J Psysom Obstet Gynecol 1993; 14 (Suppl): 45-52.

7. Verhaak CM, Smeenk JMJ, Evers AWM, Kremer JAM, Kraiimaat DDM, Braat DDM. Women's emotional adjustment to IVF: a systematic review of 25 years of research. Hum Reprod Update 2007; 13 (1): 27-36.

8. Greil AL, Shreffler KM, Schmidt L, McQuillan J. Variation in distress among women with infertility: evidence from a population-based sample. Hum Reprod 2011; 26 (8): 2101-12.

9. Boivin J, Takefman J, Braverman A. The Fertility Quality of Life (FertiQoL) tool: development and general psychometric properties. Hum Reprod 2011; 26 (8): 2084-91.

10. WHO and The World Bank 2011: World Report on Disability. Geneva: World Health Organization; 2011.

11. Boivin J, Domar AD, Shapiro DB, Wischmann TH, Fauser BCJM, Verhaak C. Tackling burden in ART: an integrated approach for medical staff. Hum Reprod 2012; 27 (4): 941-50.

12. Gameiro S, Boivin J, Peronace L, Verhaak CM. Why do patients discontinue fertility treatment? A systematic review of reasons and predictors of discontinuation in fertility treatment. Hum Reprod Update 2012; 18 (6): 652-69. 
13. Guía para el estudio y tratamiento de la infertilidad. Programa Nacional Salud de la Mujer, Departamento de Ciclo Vital, Ministerio de Salud 2015.

14. WHOQOL GROUP. The World Health Organization Quality of life assessment (WHOQOL). Position paper from the World Health Organization. Soc Sci Med 1995; 41 (10): 1403-9.

15. Schwartzmann L. Calidad de vida relacionada con la salud: aspectos conceptuales. Ciencia y Enfermería 2003; $9(2): 9-21$.

16. Testa M. Current concepts: assessment of quality of life outcomes. N Engl J Med 1996; 334 (13): 835-40.

17. Aarts JWM, Huppelschoten AG, van Empel IWH, Boivin J, Verhaak CM, Kremer JAM, et al. How patient-centered care relates to patients' quality of life and distress: a study in 427 women experiencing infertility. Hum Reprod 2012; 27 (5): 488-95.

18. Cserepes RE, Bugán A, Korösi T, Toth B, Rösner S, Strowitzki T, et al. Infertility specific quality of life and gender role attitudes in German and Hungarian involuntary childless couples. Geburtshilfe Frauenheilkd 2014; 74 (11): 1009-15.

19. Donarelli Z, Lo Coco G, Gullo S, Salerno L, Marino A, Sammartano F, et al. The Fertility Quality of Life Questionnaire (FertiQoL) Relational subscale: psychometric properties and discriminant validity across gender. Hum Reprod 2016; 31(9): 2061-71.

20. Heredia M, Tenías J, Rocio R, Amparo F, Calleja M, Valenzuela J. Quality of life and predictive factors in patients undergoing assisted reproduction techniques. Eur J Obstet Gynecol Reprod Biol 2013; 167 (2): 176-80.

21. Pedro J, Canavarro MC, Boivin J, Gameiro S. Positive experiences of patient-centred care are associated with intentions to comply with fertility treatment: Findings from the validation of the Portuguese version of the PCQ-Infertility tool. Hum Reprod 2013; 28 (9): 246272.

22. Boivin J, Scanlan LC, Walker SM. Why are infertile patients not using psychological counseling? Hum Reprod
1999; 14 (5): 1384-91.

23. Wischmann T, Korge K, Scherg H, Strowitzki T, Verres R. A 10-year follow-up study of psychosocial factors affecting couples after infertility treatment. Hum Reprod 2012; 27 (11): 3226-32.

24. Melo C, Gameiro S, Canavarro MC, Boivin J. Does the FertiQoL assess quality of life? Results from the validation of the Portuguese version of the FertiQoL. Hum Reprod 2012; 27 (suppl 2): 268-73.

25. Herrmann D, Scherg H, Verres R, Von Hagens C, Strowitzki T, Wischmann T. Resilience in infertile couples acts as a protective factor against infertility-specific distress and impaired quality of life. J Assist Reprod Genet November 2011; 28 (11): 1111-7.

26. Greil AL, Slauson-Blevins K, McQuillan J. The experience of infertility: a review of recent literature. Sociol Health Illn 2010; 32 (2): 140-62.

27. Burns LH, Covington SN. Psychology of infertility. Covington SN y Burns LH Editores: Infertility counseling: a comprehensive handbook for clinicians. Cambridge University Press. 2006, p. 1-19.

28. Newton CR, Houle M. Gender differences in psychological response to infertility treatment. Infertil Reprod Med Clin North Am 1993; 4 (3): 545-8.

29. Hammerli K, Hansjorg Z, Barth J. The efficacy of psychological interventions for infertile patients: a meta-analysis examining mental health and pregnancy rate. Hum Reprod Update 2009; 15 (3): 279-95.

30. Van der Broek U, Holvoet L, Enzlin P, Bakelants E, Demyttenaere K, D’Hooghe T. Reasons for dropout in infertility treatment. Gynecol Obstet Invest 2009; 68 (1): 58-64.

31. De Liz T, Strauss B. Differential efficacy of group and individual/couple psychotherapy with infertile patients. Hum Reprod 2005; 20 (5): 1324-32.

32. Dancet EA, Nelen WL, Sermeus W, De Leeuw L, Kremer JA, D'Hooghe TM. The patient's perspective on fertility care: a systematic review. Hum Reprod Update 2010; 16 (5): 467-87. 